Original Research Paper

\title{
On the General Solution to the Bratu and Generalized Bratu Equations
}

\author{
${ }^{1}$ Lucas Hervé Koudahoun, ${ }^{1}$ Jean Akande, ${ }^{1}$ Damien Kêgnidé Kolawolé Adjaï, \\ ${ }^{2}$ Yélomè Judicaël Fernando Kpomahou and ${ }^{1}$ Marc Delphin Monsia \\ ${ }^{I}$ Department of Physics, University of Abomey-Calavi, Abomey-Calavi, 01. BP.526, Cotonou, Benin \\ ${ }^{2}$ Department of Industrial and Technical Sciences, ENSET-Lokossa, University of Abomey, Abomey, Benin
}

Article history

Received: 12-05-2018

Revised: 09-06-2018

Accepted: 06-07-2018

Corresponding Author:

Lucas Hervé Koudahoun

Department of Physics,

University of Abomey-Calavi,

Abomey-Calavi, 01. BP.526,

Cotonou, Benin

Email: herluckou@gmail.com

\begin{abstract}
This work shows that the Bratu equation belongs to a general class of Liénard-type equations for which the general solution may be exactly and explicitly computed within the framework of the generalized Sundman transformation. In this perspective the exact solution of the Bratu nonlinear two-point boundary value problem as well as of some wellknown Bratu-type problems have been determined.
\end{abstract}

Keywords: Bratu Equation, Boundary Value Problem, Initial Value Problem, General Solution, Generalized Sundman Transformation

\section{Introduction}

The mathematical modeling of many processes in physics as in other applied disciplines of science is often achieved in terms of differential equations supplemented by initial or boundary conditions. An initial problem requires the specification of the solution at a point, while several points of specification are needed for the solution to a boundary value problem. In this regard, solving explicitly and exactly a boundary value problem becomes more mathematically complicated than a problem with initial conditions. This complication is accentuated when there is a nonlinear boundary value problem since, up to now there is no explicit and exact general method that can account for the individual behavior of each nonlinear process. The Bratu nonlinear two-point boundary value problem is one of those nonlinear problems whose explicit and exact solution for a wide variety of initial and boundary conditions remains very difficult to formulate. The Bratu problem is also one of the most investigated boundary value problems in mathematics (Ascher et al., 1994; Wazwaz, 2005). This problem derives its importance first from the combustion theory where it has been used for several applications (Wazwaz, 2016; 2005) and secondly, from the fact that its exact solution is well known (Ascher et al., 1995; Wazwaz, 2005) so that it has been widely applied to test the accuracy and efficiency of many approximate methods of different complexity like the Adomian decomposition approach (Wazwaz, 2005), the Legendre wavelet method (Venkatesh et al., 2012), the perturbation technique (Aksoy and Pakdemirli, 2010) and the virial theorem (Amore and Fernàndez, 2009). This solution exhibits also a bifurcation pattern, which only characterizes nonlinear differential equations. The one-dimensional Bratu boundary value problem may be written (He, 2006; Wazwaz, 2005):

$u^{\prime \prime}(x)+\lambda e^{u(x)}=0$

where:

$u(0)=u(1)=0$,

and $\lambda$ is a constant. The Bratu type initial value problems have also been examined by a number of authors (Venkatesh et al., 2012; Wazwaz, 2005). Such an importance motivates the reason to investigate the explicit and exact general solution to the Bratu Equation 1. The usual way to solve a boundary or initial value problem consists of computing first the general solution to the differential equation and secondly of finding the arbitrary parameters by applying the boundary or initial conditions (Bronson and Costa, 2010). So, several methods are developed in mathematics for finding explicit and exact solutions to nonlinear differential equations. In this way the variables change like the point transformation, the contact transformation and the generalized Sundman transformation may be mentioned. 
The generalized Sundman linearization theory has been recently the object of many applications so that new first integrals (Partha et al., 2010) and general periodic solutions (Akande et al., 2017; Adjaï et al., 2017) for well-known nonlinear differential equations have been computed explicitly. As such the generalized Sundman transformation recently developed by the authors of this work (Akande et al., 2017; Adjaï et al., 2017) has successfully been applied to determine the explicit and exact general periodic solutions to various types of Liénard nonlinear differential equations. In this regard a general class of quadratic Liénard type equations whose exact general solutions are trigonometric functions has been for the first time highlighted by the application of the generalized Sundman transformation under consideration (Akande et al., 2017; Adjaï et al., 2017). It has been, particularly, possible to show in this context that the well-known Painlevé-Gambier XVIII equation and its inverted version admit, for the first time, a trigonometric function as explicit and exact general periodic solution but with amplitude dependent frequency (Akande et al., 2017). This is also shown for a reduced Painlevé-Gambier XII equation under an appropriate parametric choice but with a shift factor (Adjaï et al., 2017). The same generalized Sundman transformation has been used to compute successfully the explicit and exact general periodic solutions of the famous cubic Duffing equation in terms of Jacobian elliptic functions, as expected. In spite of this progress in explicit and exact methods for solving nonlinear differential equations, it seems that a century after, the general solution to the Bratu differential Equation 1 from which the exact solution to the Bratu initial and boundary value problems may be computed by determination of integration constants, is not, unfortunately, computed in the literature explicitly and exactly in a straightforward manner (Cohen and Toledo Benavides, 2007). In this perspective, since it is almost impossible to find in the literature an explicit and exact general solution to the Bratu differential Equation 1 in a straightforward fashion, there appears convenient to examine, regarding the above, such a problem of finding the general solution by means of the generalized Sundman transformation previously mentioned (Akandeet al., 2017; Adjaï et al., 2017). In other words, within the framework of the generalized Sundman transformation under consideration, the problem of interest in this study is to ask whether such a nonlocal transformation may be successfully applied to compute the explicit and exact general solution to the Bratu differential equation, from which the exact solution to the one-dimensional Bratu boundary value problem may be deduced. More precisely, in this context, the following question may be posed: Can we compute the general solution to the Bratu Equation 1 from which the exact solution to the Bratu boundary value problem may be determined? Such a general solution is of high interest from theoretical point of view since it may allow one not only, to better understand the analytical properties of the Bratu equation under various types of initial and boundary conditions but also to detect the connection between the Bratu equation and other differential equations. From a practical point of view, it may allow the use of the Bratu equation adequately and satisfactorily as a simulation model for a large variety of engineering applications under various types of initial and boundary conditions and may serve to better test the accuracy and effectiveness of various approximation theories. In this study, it is assumed that such a general solution may be computed explicitly and exactly by the application of the generalized Sundman transformation. To demonstrate, the generalized Sundman transformation theory needed is first reviewed (section 2) and secondly the generalized Bratu equation of interest (section 3) as well as its explicit and exact general solution are established (section 4) such that the wellknown exact solution to the one-dimensional Bratu boundary value problem may be deduced (section 5). Finally the explicit and exact solutions to some Bratu type initial and boundary value problems examined by Wazwaz (2005) using Adomian decomposition method and also by Boyd (2011) are easily computed (section 6) so that a discussion of results (section 7) and a conclusion may be addressed.

\section{Review of the Generalized Sundman linearization theory}

In this section the generalized Sundman linearization theory recently introduced by Akande et al. (2017) is considered. The application of this linearizing transformation requires to consider the general class of quadratic Liénard type nonlinear differential equations:

$$
\begin{aligned}
& u^{\prime \prime}(x)+\left(\ell \frac{g^{\prime}(u)}{g(u)}-\gamma \varphi^{\prime}(u)\right) u^{\prime 2}(x) \\
& +\frac{a^{2} \exp (2 \gamma \varphi(u)) \int g(u)^{\ell} d u}{g(u)^{\ell}}=0
\end{aligned}
$$

which may be reduced under the conditions:

$$
\begin{aligned}
& y(\tau)=F(x, u), d \tau=G(x, u) d x, \\
& G(x, u) \frac{\partial F(x, u)}{\partial u} \neq 0
\end{aligned}
$$

with:

$$
F(x, u)=\int g(u)^{\ell} d u, \quad G(x, u)=\exp (\gamma \phi(u))
$$


to

$$
y^{\prime \prime}(\tau)+a^{2} y(\tau)=0
$$

The Equation 5 admits the solution:

$$
y(\tau)=A_{0} \sin (a \tau+\alpha)
$$

where prime denote ordinary differentiation of the dependent variable with respect to the argument, $A_{0}, \alpha, a$, $\ell$ and $\gamma$ are arbitrary parameters. The functions $\varphi(u)$ and $g(u) \neq 0$, are arbitrary functions of $u$. So with that the generalized Bratu equation of interest may be established.

\section{Generalized Bratu Equation}

This section is devoted to carry out the generalized Bratu equation under question. To that end it is required that:

$$
\ell \frac{g^{\prime}(u)}{g(u)}-\gamma \varphi^{\prime}(u)=0, \ell \neq 0
$$

that is:

$$
g(u)=e^{\frac{\gamma}{\ell} \varphi(u)}
$$

such that (3) becomes:

$$
u^{\prime \prime}(x)+a^{2} e^{\gamma \varphi(u)} \int e^{\gamma \varphi(u)} d u=0
$$

The application of $\varphi(u)=u$, to (9) yields as equation:

$$
u^{\prime \prime}(x)+\frac{a^{2}}{\gamma} e^{2 \gamma u(u)}=0
$$

The above shows the following theorem.

\section{Theorem 1}

Let $\varphi(u)=u$ and $g(u)=e^{\frac{\gamma}{\ell} u}$. Then Equation 3 reduces to $(10)$.

The Equation 10 is the desired generalized Bratu equation.

\section{Theorem 2}

Let $\gamma=\frac{1}{2}$ and $2 a^{2}=\lambda$. Then (Equation 10) is reducible to (Equation 1).

\section{Proof}

The theorem 2 is a consequence of theorem 1 , that is a particular case $\left(\gamma=\frac{1}{2}\right.$ and $\left.2 a^{2}=\lambda\right)$ of theorem 1 . To observe this, it suffices to introduce $2 \gamma=1$ and $2 a^{2}=\lambda$, into (Equation 10) to obtain (He, 2006; Wazwaz, 2005) immediatly the celebrated Bratu Equation 1. In this regard the explicit and exact general solution in question may, as one can see, be easily computed for various boundary and initial conditions, in other words for all $x \in I R$.

\section{General Solutions}

In this section the general solutions to the Bratu equation and generalized Bratu Equation 10 are explicitly and exactly computed under the conditions (4), such that one may deduce the well-known exact solution to the onedimensional Bratu boundary value problem. Thus substituting Equation 8 into 4 leads to:

$$
y(\tau)=\frac{1}{\gamma} e^{\gamma u}
$$

so that the general solution becomes:

$$
u(x)=\frac{1}{\gamma} \ln (\gamma y(\tau))
$$

Knowing (Equation 6), Equation 12 may be written as:

$$
u(x)=\frac{1}{\gamma} \ln \left(\gamma A_{0} \sin (a \tau+\alpha)\right)
$$

The problem is now to express $\sin (a \tau+\alpha)$ in terms of $x$. In this way the preceding relation $d \tau=\exp (\gamma \varphi(u)) d x$ reduces to:

$$
\frac{d \tau}{A_{0} \sin (a \tau+\alpha)}=\gamma d x
$$

that is:

$$
\frac{d \tau}{\sin (a \tau+\alpha)}=A_{0} \gamma d x
$$

such that:

$a \tau+\alpha=2 \operatorname{tg}^{-1}\left(K e^{\gamma a A_{0} x}\right)$

where, $K>0$ is an arbitrary constant. In this context the general solution (13) may take the form: 


$$
u(x)=\frac{1}{\gamma} \ln \left\{\gamma A_{0} \sin \left[2 \operatorname{tg}^{-1}\left(K e^{\gamma a A_{0} x}\right)\right]\right\}
$$

where, $\gamma>0$. The Equation 16 is the desired explicit and exact general solution to the generalized Bratu Equation 10 for all $x \in I R$. Therefore the following theorem results from the above.

\section{Theorem 3}

Equation 10 has the solution (Equation 16) if and only if (Equation 5) has the solution (Equation 6).

The parametric choice $\gamma=\frac{1}{2}$, yields the general solution under question to the Bratu Equation 1, that is:

$$
u(x)=2 \ln \left\{\frac{A_{0}}{2} \sin \left[2 \operatorname{tg}^{-1}\left(K e^{\frac{a A_{0}}{2} x}\right)\right]\right\}
$$

for all $x \in I R$. As a consequence of the above, the following theorem may be formulated.

\section{Theorem 4}

$$
\text { Let } \gamma=\frac{1}{2} \text {. Then Equation } 16 \text { becomes (Equation 17). }
$$

In this context the integration constants $K$ and $A_{0}$ may be determined for various initial and boundary conditions. In other words, the behavior of $u(x)$ depends on these conditions. The objective is now to show that the general solution (Equation 17) may yield the wellknown exact solution to the one-dimensional Bratu boundary value problem under the conditions that $u(0)=$ $u(1)=0$.

\section{Exact Solution to the Bratu Boundary Value Problem}

This section is devoted to determine the exact solution of the one-dimensional Bratu boundary value problem, in other words to compute the two constants of integration $A_{0}$ and $K$ under the conditions that $u(0)=u(1)$ $=0$. So the application of $u(0)=0$ leads to:

$$
\ln \left\{A_{0} \gamma \sin \left(2 \operatorname{tg}^{-1} K\right)\right\}=0
$$

that is:

$$
\sin \left(2 \operatorname{tg}^{-1} K\right)=\frac{1}{A_{0} \gamma}
$$

Knowing that:

$$
\sin \left(2 t g^{-1} K\right)=\frac{2 K}{1+K^{2}}
$$

the Equation 18 becomes:

$$
\frac{2 K}{1+K^{2}}=\frac{1}{A_{0} \gamma}
$$

On the other hand the application of the condition $u(1)=0$, gives:

$$
\ln \left\{A_{0} \gamma \sin \left\lfloor 2 \operatorname{tg}^{-1}\left(K e^{\gamma a A_{0}}\right)\right\rfloor\right\}=0
$$

that is:

$\sin \left[2 \operatorname{tg}^{-1}\left(K e^{\gamma a A_{0}}\right)\right]=\frac{1}{A_{0} \gamma}$

which may be written in the form:

$\frac{2 K e^{\gamma a A_{0}}}{1+K^{2} e^{2 \gamma a A_{0}}}=\frac{1}{A_{0} \gamma}$

Equating (Equation 20) and (Equation 22) yields:

$\frac{2 K e^{\gamma a A_{0}}}{1+K^{2} e^{2 \gamma a A_{0}}}=\frac{2 K}{1+K^{2}}$

which leads after a little mathematical treatment to:

$K=e^{-\frac{a A_{0} \gamma}{2}}$

In this context the parameter $A_{0}$ may be computed, using (Equation 20) as:

$A_{0}=\frac{1}{\gamma} \cosh \left(\frac{a \gamma A_{0}}{2}\right)$

such that the general solution (Equation 16), that is, the exact solution to the generalized Bratu Equation 10 under the boundary conditions $u(0)=u(1)=0$, may take the expression:

$u(x)=\frac{1}{\gamma} \ln \left\{\frac{2 \gamma A_{0} e^{a \gamma A_{0}\left(x-\frac{1}{2}\right)}}{1+e^{2 a \gamma A_{0}\left(x-\frac{1}{2}\right)}}\right\}$

or:

$u(x)=-\frac{1}{\gamma} \ln \left\{\frac{1+e^{2 a \gamma A_{0}\left(x-\frac{1}{2}\right)}}{2 \gamma A_{0} e^{a \gamma A_{0}\left(x-\frac{1}{2}\right)}}\right\}$

Using the identity: 


$$
\cosh q=\frac{e^{-q}}{2}\left(1+e^{2 q}\right)
$$

the Equation 27 may be expressed as:

$$
u(x)=-\frac{1}{\gamma} \ln \left\{\frac{\cosh \left[a \gamma A_{0}\left(x-\frac{1}{2}\right)\right]}{\cosh \left(\frac{a \gamma A_{0}}{2}\right)}\right\}
$$

The expression (Equation 29) is the desired explicit and exact solution to the generalized Bratu Equation 10 under the boundary conditions in consideration. In this situation the above results show the following theorem.

\section{Theorem 5}

Consider (Equation 2). Then (Equation 16) reduces to (Equation 29).

So the exact solution of the one-dimensional Bratu boundary value problem may, for the value $\gamma=\frac{1}{2}$, take the expression:

$$
u(x)=-2 \ln \left\{\frac{\cosh \left[\frac{a A_{0}}{2}\left(x-\frac{1}{2}\right)\right]}{\cosh \left(\frac{a A_{0}}{4}\right)}\right\}
$$

so that for the parametric choice:

$$
\theta=a A_{0}
$$

that is for the transcendental equation:

$$
\theta=2 a \cosh \left(\frac{\theta}{4}\right)
$$

the exact solution to the Bratu boundary value problem may be definitively written as:

$$
u(x)=-2 \ln \left\{\frac{\cosh \left[\frac{\theta}{2}\left(x-\frac{1}{2}\right)\right]}{\cosh \left(\frac{\theta}{4}\right)}\right\}
$$

Therefore as a special case of the theorem 5 , the following result may be expressed.

\section{Theorem 6}

$$
\text { Let } \gamma=\frac{1}{2} \text { and } \theta=2 a \cosh \left(\frac{\theta}{4}\right) \text {. Then (Equation 29) is }
$$
reducible to (Equation 32).
After showing that the exact solution of the onedimensional Bratu boundary value problem may be calculated from the general solution to the Bratu differential equation, the purpose, now, is to show that the current general theory may also be used to compute the explicit and exact solutions to the initial and boundary value problems investigated by Wazwaz (2005) on the basis of Adomian decomposition method (Wazwaz, 2005) and Boyd (2011).

\section{Bratu Type Initial and Boundary Value Problems}

In the investigation of the Bratu boundary value problem by Adomian decomposition method, Wazwaz (2005) considered a number of Bratu type initial and boundary value problems. The results obtained by Wazwaz (2005) are later used by several authors (Venkatesh et al., 2012; Aksoy and Pakdemirli, 2010) to test the accuracy and efficiency of some approximate methods for solving differential equations. In this section, the explicit and exact solutions of the Bratu type boundary value problem investigated by Boyd (2011) and the Bratu type initial value problem considered by Wazwaz (2005) are determined using the general solution established in this study.

\section{Bratu-Type Problem 1}

The Bratu-type problem considered by Boyd (2011) may be written in the form:

$$
\begin{aligned}
& u^{\prime \prime}(x)+\lambda e^{u(x)}=0,-1<x<1 \\
& u(-1)=u(1)=0
\end{aligned}
$$

Although, here, the differential equation is that of Bratu, the boundary conditions are different from those usually used for the Bratu nonlinear two-point boundary value problem. In this regard under the condition $u(-1)=$ 0, the general solution (Equation 16) yields:

$$
\ln \left\{A_{0} \gamma \sin \left[2 \operatorname{tg}^{-1}\left(K e^{-\gamma a A_{0}}\right)\right]\right\}=0
$$

that is:

$$
\sin \left[2 \operatorname{tg}^{-1}\left(K e^{-\gamma a A_{0}}\right)\right]=\frac{1}{A_{0} \gamma}
$$

which may be reduced to:

$$
\frac{2 K e^{-\gamma a A_{0}}}{1+K^{2} e^{-2 \gamma a A_{0}}}=\frac{1}{A_{0} \gamma}
$$

On the other hand, the application of $u(1)=0$, turns (Equation 16) into: 


$$
\ln \left\{A_{0} \gamma \sin \left[2 \operatorname{tg}^{-1}\left(\operatorname{Ke}^{\gamma A_{0}}\right)\right]\right\}=0
$$

which leads to:

$$
\sin \left[2 \operatorname{tg}^{-1}\left(K e^{\gamma A_{0}}\right)\right]=\frac{1}{A_{0} \gamma}
$$

such that:

$$
\frac{2 K e^{\gamma / A_{0}}}{1+K^{2} e^{2 \gamma a_{0}}}=\frac{1}{A_{0} \gamma}
$$

The comparison of (Equation 35) with (Equation 37) allows, after a few mathematical treatments, to obtain:

$$
K=1
$$

so that the second integration constant $A_{0}$ may take the form:

$$
A_{0}=\frac{1}{2 \gamma}\left(e^{-a \gamma A_{0}}+e^{a \gamma A_{0}}\right)
$$

which may also be written:

$$
A_{0}=\frac{1}{\gamma} \cosh \left(a \gamma A_{0}\right)
$$

In this context the general solution (Equation 16) becomes:

$$
u(x)=\frac{1}{\gamma} \ln \left\{\gamma A_{0} \sin \left[2 \operatorname{tg}^{-1}\left(e^{\gamma a_{0} x}\right)\right]\right\}
$$

where, $A_{0}$ is given by (Equation 39). Knowing that (Equation 1) is obtained for $\gamma=\frac{1}{2}$, the exact solution to the boundary value problem (Equation 33 ) becomes:

$$
u(x)=2 \ln \left\{\frac{A_{0}}{2} \sin \left[2 \operatorname{tg}^{-1}\left(e^{\frac{a A_{0}}{2} x}\right)\right]\right\}
$$

where:

$$
A_{0}=2 \cosh \left(\frac{a A_{0}}{2}\right)
$$

Using the identity:

$$
\sin \left[2 \operatorname{tg}^{-1}\left(e^{\frac{a A_{0}}{2} x}\right)\right]=\frac{1}{\cosh \left(\frac{a A_{0}}{2} x\right)}
$$

the solution (Equation 41) takes the form:

$$
u(x)=2 \ln \left\{\frac{2}{A_{0}} \cosh \left(\frac{a A_{0}}{2} x\right)\right\}
$$

that is:

$$
u(x)=-2 \ln \left\{\frac{\cosh \left(\frac{a A_{0}}{2} x\right)}{\cosh \left(\frac{a A_{0}}{2}\right)}\right\}
$$

where, $A_{0}$ is given by (Equation 42). Now, a few algebraic manipulations is needed to compare the exact solution (Equation 44) to the problem (Equation 33) with the solution given by Boyd (2011). As $2 a^{2}=\lambda$, that is $a= \pm \sqrt{\frac{\lambda}{2}}$, it suffices to set $\frac{A_{0}}{2}=z$, which is equivalent to $\cosh \left(\frac{A_{0}}{2} \sqrt{\frac{\lambda}{2}}\right)=z$, that is to say $\cosh \left(z \sqrt{\frac{\lambda}{2}}\right)=z$, to write (Equation 44) in the form:

$u(x)=-2 \ln \left\{\frac{\cosh \left(z \sqrt{\frac{\lambda}{2}} x\right)}{\cosh \left(z \sqrt{\frac{\lambda}{2}}\right)}\right\}$

or definitively under the expression:

$u(x)=\ln \left\{z^{2} \sec h^{2}\left(z \sqrt{\frac{\lambda}{2}} x\right)\right\}$

which is nothing but the form used by Boyd (2011) to express the solution to the boundary value problem (Equation 33).

\section{Bratu-Type Problem 2}

The third Bratu-type problem solved by Wazwaz (2005) is an initial value problem formulated as:

$u^{\prime \prime}(x)-2 e^{u(x)}=0,0<x<1$
$u(0)=u^{\prime}(0)=0$

It is convenient before solving (Equation 47) to consider the general solution (Equation 16) under the general initial conditions $u(0)=u_{0}$ and $u^{\prime}(0)=v_{0}$. In this perspective, the application of $u(0)=u_{0}$, gives:

$e^{\gamma u_{0}}=\gamma A_{0} \sin \left(2 \operatorname{tg}^{-1} K\right)$ 
and the application of $u^{\prime}(0)=v_{0}$, leads to:

$v_{0}=\frac{2 a A_{0} K \cos \left(2 \operatorname{tg}^{-1} K\right)}{\left(1+K^{2}\right) \sin \left(2 \operatorname{tg}^{-1} K\right)}$

so that (49) may be rewritten as:

$v_{0}=\frac{2 \gamma a A_{0}^{2} K \cos \left(2 \operatorname{tg}^{-1} K\right)}{\left(1+K^{2}\right) e^{\gamma u_{0}}}$

Therefore, for the initial conditions $u_{0}=0$ and $v_{0}=0$, one may find $K=1$ and $A_{0}=2$, such that the general solution (Equation 16) under the above conditions reduces to:

$u(x)=\frac{1}{\gamma} \ln \left\{2 \gamma \sin \left[2 \operatorname{tg}^{-1}\left(e^{2 a \gamma x}\right)\right]\right\}$

As the Bratu type initial value problem (Equation 47) is obtained from (10) for $\gamma=\frac{1}{2}$ and $a^{2}=-1$, the exact solution to (Equation 47) may take the expression:

$u(x)=2 \ln \left\{\sin \left[2 \operatorname{tg}^{-1}\left(e^{i x}\right)\right]\right\}$

where, $i$ is the purely imaginary number.

Using the identity:

$\sin \left[2 \operatorname{tg}^{-1}\left(e^{i x}\right)\right]=\frac{1}{\cosh (i x)}$

the solution (52) may be written as:

$u(x)=-2 \ln (\cosh (i x))$

which takes the definitive expression:

$u(x)=-2 \ln (\cos (x))$

This result (Equation 55) is identical to that obtained by Wazwaz using the Adomian decomposition method (Wazwaz, 2005).

\section{Discussion}

Although the Bratu boundary value problem has been intensively investigated in the literature, one may unfortunately note that there doesn't exist an explicit and exact general solution to the differential equation which describes this problem. In this study the Bratu equation is investigated with the aim of finding general solution by using an explicit and exact method for solving nonlinear differential equations such that various initial and boundary conditions may be applied. A straightforward way to do so was to transform the Bratu nonlinear differential equation into a linear second order differential equation with well known properties. It is found in this regard that the generalized Sundman linearization theory recently developed by Akande et al. (2017) is well convenient to solve this problem. In so doing it has been highlighted that the Bratu equation consists of a special case of a generalized nonlinear differential equation admitting an explicit and exact general solution for all $x \in I R$ so that various initial and boundary conditions may be applied. In such a situation, it is, for the first time, shown that the well-known exact solution to the one-dimensional Bratu boundary value problem may be, by applying the required boundary conditions, deduced from the explicit and exact general solution to the differential equation. In this perspective, it has been possible to compute the exact solutions to some Bratu type initial and boundary value problems examined by Boyd (2011) and Wazwaz (2005) using Adomian decomposition method (Wazwaz, 2005), in the context of the current general theory. The method applied in this study shows as an advantage the fact that the Bratu nonlinear differential equation is intimately and directly related, for the first time, to the linear harmonic oscillator differential equation with well known exact analytical solution. So from the above a conclusion may be formulated for the work.

\section{Conclusion}

While the exact solution of the one-dimensional Bratu boundary value problem is well known in the literature, the explicit and exact general solution of the Bratu nonlinear differential equation is unfortunately, after a century, an unresolved question. This constitutes a fundamental drawback in the understanding of analytical properties of Bratu equation. Fortunately this shortcoming has been, for the first time, overcome in this study, using the generalized Sundman transformation, which closely relates the Bratu differential equation to the linear harmonic oscillator equation. In so doing, it was possible to deduce from the computed general solution the well-known exact solution of the Bratu boundary value problem and to show that the Bratu equation is a special case of a more general equation. Therefore, one could compute the explicit and exact solutions of a large variety of Bratu type problems with a relative simplicity.

\section{Author's Contributions}

All authors contributed to the development and formulation of this work. All authors read and approved the final manuscript for publication. 


\section{Ethics}

The authors declare that there exists no competing interests.

\section{References}

Adjaï, D.K.K., L.H. Koudahoun, J. Akande, Y.J.F. Kpomahou and M.D. Monsia, 2017. Solutions of the Duffing and Painlevé-Gambier equations by generalized Sundman transformation. viXra: $1705.0262 \mathrm{~V} 3$.

Akande, J., D.K.K. Adjaï, L.H. Koudahoun, Y.J.F. Kpomahou and M.D. Monsia, 2017. Theory of exact trigonometric periodic solutions to quadratic Liénard type equations. viXra:1704.0199V3.

Aksoy, Y. and M. Pakdemirli, 2010. New perturbationiteration solutions for Bratu-type equations. Comput. Math. Applic., 59: 2802-2808.

DOI: $10.1016 /$ j.camwa.2010.01.050

Amore, P. and F.M. Fernàndez, 2009. The, virial theorem for nonlinear problems. arXiv: 0904.3858V2.

Ascher, U.M., R.M.M. Mattheij and R.D. Russell, 1994. Numerical Solution of Boundary Value Problems for Ordinary Differential Equations. 1st Edn., SIAM, Philadelphia, ISBN-10: 1611971233, pp: 595.

Boyd, J.P., 2011. One-point pseudospectral collocation for the one-dimensional Bratu equation. Applied Math. Comput., 217: 5553-5565.

DOI: $10.1016 /$ j.amc.2010.12.029
Bronson, R. and G.B. Costa, 2006. Schaum's Outline of Differential Equations. 3rd Edn., McGraw-Hill, New York, ISBN-10: 0071456872, pp: 385.

Cohen, N. and J.V. Toledo Benavides, 2007. Explicit radial Bratu solutions in dimensional $\mathrm{n}=1$. Universidade Estadual de Campinas.

He, J.H., 2006. Some asymptotic methods for strongly nonlinear equations. Int. J. Modern Phys. B, 20: 1141-1199. DOI: 10.1142/S0217979206033796

Partha, G., B. Khanra and A.G. Choudoury, 2010. On generalized Sundman transformation method, first integrals, symmetries and solutions of equations of Painlevé-Gambier type. Nonlinear Anal., 72: 3247-3257. DOI: 10.1016/j.na.2009.12.004

Venkatesh, S.G., S.K. Ayyaswamy and S. Raja Balachandar, 2012. The Legendre wavelet method for solving initial value problems of Bratu-type. Comput. Math. Applic., 63: 1287-1295. DOI: 10.1016/j.camwa.2011.12.069

Wazwaz, A.M., 2005. Adomian decomposition method for a reliable treatment of the Bratu-type equations. Applied Math. Comput., 166: 652-663. DOI: 10.1016/j.amc.2004.06.059

Wazwaz, A.M., 2016. The successive differentiation method for solving Bratu equation and Bratu-type equations. Rom. J. Phys., 61: 774-783. 